\title{
The complexity of drug hypersensitivity reactions
}

\author{
María José Torres ${ }^{1}$ \\ ${ }^{1}$ Universidad de Málaga Facultad de Medicina
}

July 31,2020

Drug hypersensitivity reactions (DHRs) represent a global threat to healthcare systems due to their incidence, with a significant increase over last years ${ }^{1}$. DHR figures are overestimated in the general population since less than $40 \%$ of cases initially labelled as allergic can be confirmed as such when evaluated in an allergy unit ${ }^{2}$. Achieving an accurate diagnosis is complex and time consuming; besides, tests must be tailored to specific clinical manifestations and underlying mechanisms and will depend on the culprit drugs. Diagnosis often requires performing drug provocation tests (DPTs), which are especially problematic for severe reactions, making management of these patients challenging and expensive for the health care system.

Clinically, DHRs are classified into immediate and non-immediate, based on the time interval between drug exposure and onset of the symptoms ${ }^{3}$. The most severe immediate reaction is anaphylaxis. This issue of the journal has been dedicated o drug hypersensitivity, which is becoming a major public health issue during the last decade, especially with the introduction of biologicals to medicine. Bilo et al. ${ }^{4}$ evaluated the anaphylaxis mortality rate in Italy from 2004 to 2016 and found an average mortality rate for definite anaphylaxis (ICD10 code) of 0.51 per million population per year, mostly due to the use of medications (73.7\%), although in $98 \%$ of the cases culprit drugs were not identified. Regarding non-immediate reactions, one of the most challenging diagnoses is drug reaction with eosinophilia and systemic symptoms (DRESS), which is sometimes difficult, at an early stage, due to overlapping clinical symptoms with maculopapular exanthema (MPE). Pedruzzi et al. ${ }^{5}$ identified 7 microRNAs (miRNAs) that correctly classified DRESS or MPE patients and were associated with keratinocyte differentiation/skin inflammation, type I IFN pathway viral replication, ATP-binding cassette transporters, and $\mathrm{T}$ lymphocyte polarisation, being all of them potential biomarkers. Non-immunologically mediated adverse reactions, such as attention-deficit/hyperactivity disorder (ADHD) are reported by Fuhrmannet al. ${ }^{6}$ in association with systemic H1-antihistamines administration in school-age children, especially the $1^{\text {st }}$ generation agents.

The mechanism underlying DHR and the reason why patients treated with the same drug develop a tolerance response or an immediate or non-immediate DHR is not completely understood (Figure 1). Therefore, the prediction of who may experience a DHR, and if so, in what form, remains clinically obscure for most drugs. Goh SJR et al. ${ }^{7}$ elegantly analyse this complexity, using non-immediate reactions to penicillins as a model. They focus on the understanding of the role of nature of the lesional $\mathrm{T}$ cells, the characterisation of drugresponsive $\mathrm{T}$ cells isolated from patient blood, and the potential mechanisms by which penicillins enter the antigen-processing and presentation pathway to stimulate these deleterious responses.

Regarding specific drugs involved in allergy, betalactam antibiotics (BL) are the most frequent culprit, being many reactions mediated by $\operatorname{IgE}$. This type of reaction varies among patients, with some reacting only to one BL and others to several of them; it tends to change over time and differs between European countries, depending on BL consumption. Nowadays, amoxicillin (AX), alone or in combination with the $\beta$-lactamase inhibitor clavulanic acid (CLV), is the most often prescribed BL worldwide (Figure 2) and the most common elicitor of reactions in both children and adults. It is unclear why patients after the administration of AX-CLV develop selective hypersensitivity to AX, while tolerating CLV and vice-versa. Ariza et al. ${ }^{8}$ generated drugspecific T-cell clones from AX- or CLV-selective immediate hypersensitivity patients and found that both 
AX- and CLV-specific clones were generated irrespective of whether AX or CLV was the culprit, although a higher secretion of Th2 cytokines (IL-13 and IL-5) was detected when clones were activated with the culprit BL compared with clones stimulated with the tolerated BL, in which higher secretion of Th1 cytokines $(\mathrm{IFN}-\gamma)$ was observed. Regarding selective non-immediate reactions to CLV, Copaescu A et al. ${ }^{9}$ report on a cohort of patients with a history of non-immediate reaction to CLV, who demonstrated a delayed intradermal skin test response to CLV, $17 \%$ were allergic to both CLV and ampicillin, and $83 \%$ were selective reactors with good tolerance to $\mathrm{AX}$. IFN- $\gamma$ release enzyme-linked immunospot (ELISpot) was performed giving a sensitivity of $33 \%$. Other drugs such as sulphonamides, either antibiotic or non-antibiotics are important triggers of non-immediate DHRs. Vilchez-Sanchez et al. ${ }^{10}$ showed that lymphocyte transformation tests (LTT) can help avoid the performance of DPT with a sensitivity of $75 \%$, a specificity of $100 \%$, and negative and positive predictive values of $72.7 \%$ and $100 \%$, respectively.

There has been a great expansion in the use of biological agents (mainly monoclonal antibodies (mAbs)), and they have greatly improved the treatment landscape of hemato-oncologic, autoimmune, inflammatory and rheumatologic diseases. In parallel, the incidence rate of reported DHRs associated with these products has increased considerably within the last years, ranging from mild to life-threatening. Yang BC et al. ${ }^{11}$ recommend risk stratification as the first step for managing patients with DHRs to these drugs. In cases with negative skin test and mild reactions, DPT is an option, and in moderate or severe reactions, desensitisation becomes the preferred approach. In cases with positive skin test, desensitisation is the recommended course of action, especially when there is no alternative medication. Desensitisation is also widely used in the management of immediate hypersensitivity reactions to chemotherapy agents, such as platinums. There is suspicion about the presence of a longer memory of tolerance in subsequent desensitisation protocols partially resembling the regulatory tolerance mechanisms induced by allergen immunotherapy. Tüzer et al.

12 demonstrate the possible role of IL-10 in desensitisation with platinums, as they found a dynamic change in serum IL-10 levels observed as an increase during desensitisation and a decrease in between the protocols.

Finally, a wide spectrum of drugs has been considered for treatment of coronavirus disease 2019 (COVID19) and all of them can potentially induce DHRs. Gelincik A et al.$^{13}$ reviewed DHRs in COVID-19 times to these drugs, with knowledge mainly coming from previous clinical experience in patients not infected with COVID-19. As in other viral infections, skin symptoms, including exanthemas, may appear during the evolution of the disease, leading to differential diagnosis with DHRs. Whether COVID-19 can aggravate $\mathrm{T}$-cell mediated DHRs reactions as some viruses is at present unknown.

We can conclude that new drugs are continuously introduced into the markets and confirmed as inducers of hypersensitivity reactions. We still do not completely understand the mechanisms underlying many of these reactions and further studies for improving diagnostic and management are needed even in classic and well-studied drugs as BLs.

Abbreviations: AX: Amoxicillin; CLV: Clavulanic acid; COVID-19: Coronavirus disease 2019; DHR: Drug hypersensitivity reactions; DPT: Drug provocation tests; DRESS: Drug reaction with eosinophilia and systemic symptoms; ELISpot: enzyme-linked immunospot; LTT: Lymphocyte transformation tests; MPE: Maculopapular exanthema.

\section{References}

1. Mayorga C, Fernandez TD, Montanez MI, Moreno E, Torres MJ. Recent developments and highlights in drug hypersensitivity. Allergy 2019;74 (12):2368-2381.

2. Romano A, Atanaskovic-Markovic M, Barbaud A, Bircher AJ, Brockow K, Caubet JC, et al. Towards a more precise diagnosis of hypersensitivity to beta-lactams - an EAACI position paper. Allergy 2020;75 (6):1300-1315.

3. Pichler WJ. Immune pathomechanism and classification of drug hypersensitivity. Allergy2019;74 (8):14571471. 
4. Bilo MB, Corsi A, Martini M, Penza E, Grippo F, Bignardi D. Fatal anaphylaxis in Italy: Analysis of cause-of-death national data, 2004-2016. Allergy 2020.

5. Pedruzzi E, Chasset F, Duroux-Richard I, Bocarra D, Apparailly F, Nourikyan J, et al. Dysregulation of microRNA expression in the skin during cutaneous adverse drug reactions. Allergy 2020.

6. Fuhrmann S, Tesch F, Romanos M, Abraham S, Schmitt J. ADHD in school-age children is related to infant exposure to systemic H1-antihistamines. Allergy 2020.

7. Goh SJR, Tuomisto JEE, Purcell AW, Mifsud NA, Illing PT. The complexity of T cell-mediated penicillin hypersensitivity reactions. Allergy 2020.

8. Ariza A, Fernandez-Santamaria R, Meng X, Salas M, Ogese MO, Tailor A, et al. Characterization of amoxicillin and clavulanic acid specific T-cell clones from patients with immediate drug hypersensitivity. Allergy 2020.

9. Copaescu A, Rose M, Mouhtouris E, Chua KY, Holmes NE, Phillips EJ, et al. Delayed hypersensitivity associated with amoxicillin-clavulanate. Allergy 2020.

10. Vilchez-Sanchez F, Loli-Ausejo D, Rodriguez-Mariblanca A, Montserrat-Villatoro J, Ramirez E, Dominguez-Ortega J, et al. Lymphocyte transformation test can be useful for the diagnosis of delayed adverse reactions to sulfonamides. Allergy 2020.

11. Yang BC, Castells M. Diagnosis and treatment of drug hypersensitivity reactions to biologicals: Medical algorithm. Allergy 2020.

12. Tuzer C, Sari M, Aktas Cetin E, Ak N, Buyukozturk S, Colakoglu B, et al. Rapid drug desensitization for platinum-based chemotherapy drugs significantly increases peripheral blood IL-10 levels. Allergy 2020.

13. Gelincik A, Brockow K, Celik GE, Dona I, Mayorga C, Romano A, et al. Diagnosis and management of the drug hypersensitivity reactions in Coronavirus disease 19. Allergy2020.

Figure 1.- Different mechanisms of hypersensitivity reactions induced by drugs. Drugs or their metabolites interact with the antigen-presenting cells (APC), dendritic cells (DC) that depending on the maturational or activation status will present the drug to T-lymphocytes in lymph nodes inducing immediate, delayed or tolerant drug-specific immune responses.

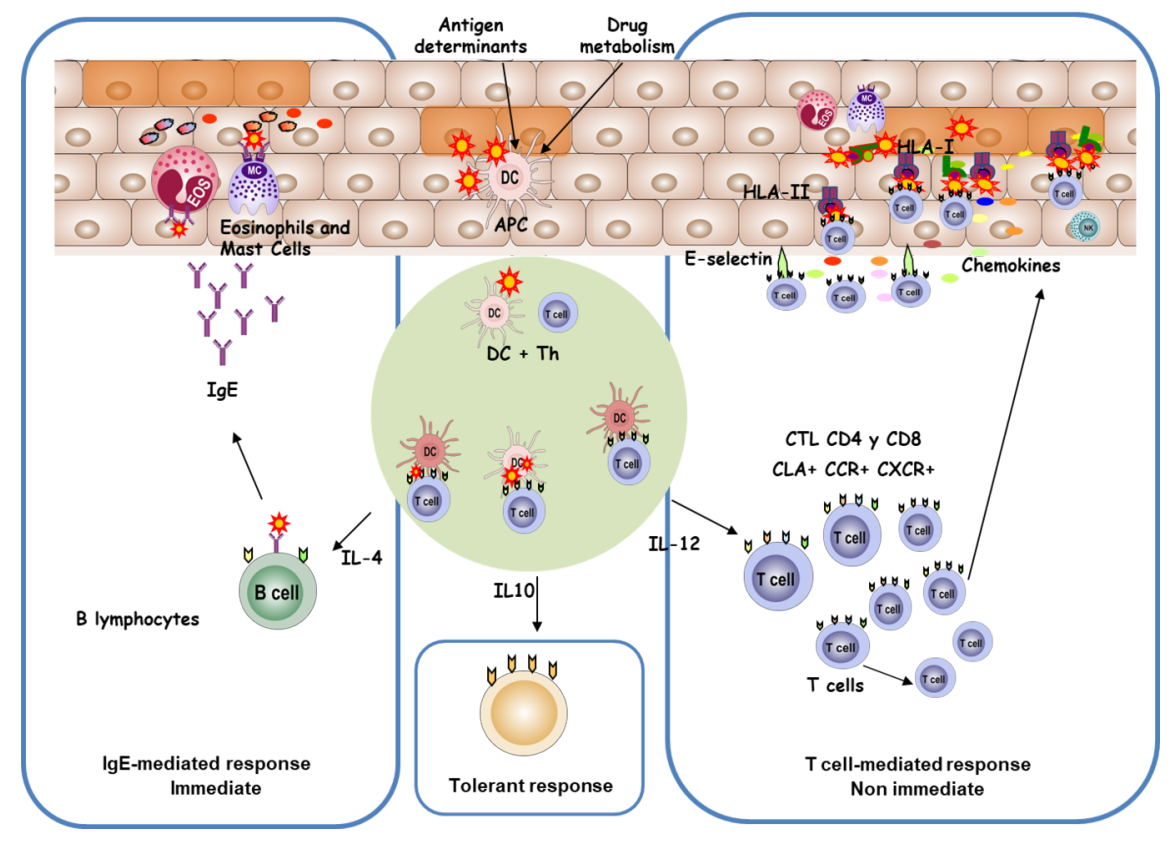


Figure 2.- Betalactam comsumption (Data obtained from Surveillance of antimicrobial consumption in Europe 2012. In ECDC Surveillance Report; European Centre for Disease Prevention and Control (ECDC): Stockholm: ECDC, 2014, p 73; and Versporten, A.et al. J Antimicrob Chemother 2011, 66 Suppl 6, vi13-23.)
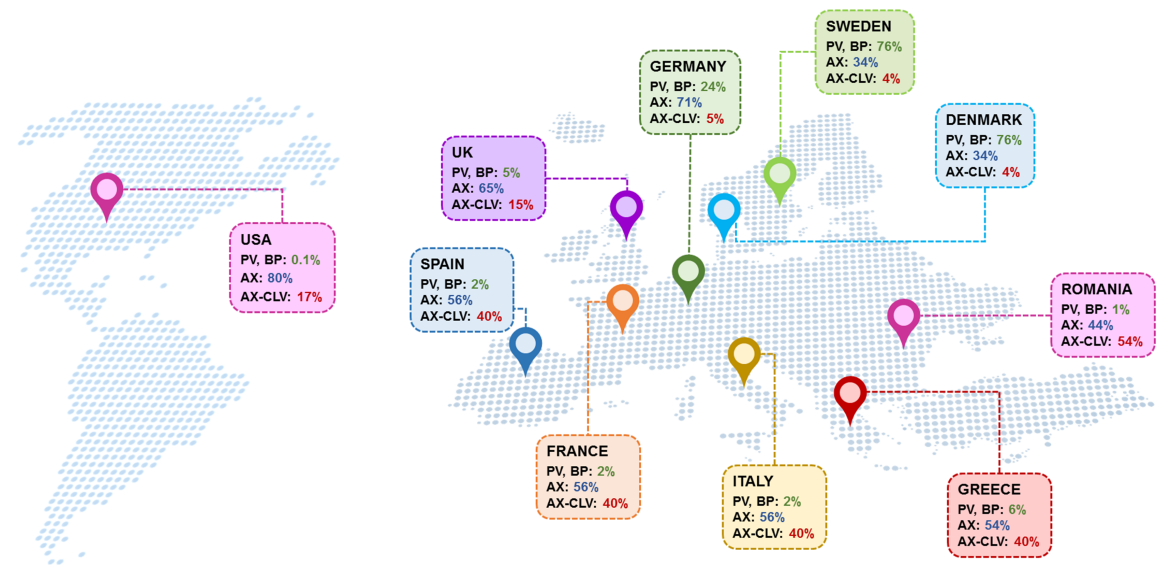